\section{Conhecimento e prática sobre os fatores de risco para o câncer de mama entre mulheres de 40 a 69 anos}

\section{Knowledge of and practices regarding risk factors for breast cancer in women aged between 40 and 69 years}

Adriane Pires Batiston 1

Edson Mamoru Tamaki 2

Laís Alves de Souza 3

Mara Lisiane de Moraes dos Santos 4

\begin{abstract}
Objectives: to investigate knowledge of and practices regarding risk factors for breast cancer among users of the Family Health Strategy (FHS).

Methods: a cross-sectional study was carried out among 393 women aged between 40 and 69 years using the FHS in the city of Dourados, in the Brazilian State of Mato Grosso do Sul. An interview was conducted using a semi-structured questionnaire to investigate socio-demographic variables, family history and awareness/practices regarding the risk factors for breast cancer. The variables were described using simple frequency and a percentage. The association between awareness of the risk factors and the variables was confirmed using Fisher's exact test and the chisquare with a level of significance of $5 \%$.

Results: the mean age was $52.5 \pm 8.1$ years, the mean years of schooling was $4.4 \pm 3.6$ years, $52.4 \%$ of the women were black/colored and $66.6 \%$ had a partner. Of the women, $86.5 \%$ had received some information on breast cancer. The risk factors for the disease are known by $54.2 \%$ of the women. Awareness of the risk factors was associated with family history $(p=0.004)$ and years of schooling $(p=0.01)$. Where the risk factors were known, $52.2 \%$ of the women took preventive measures.

Conclusions: the identification of variables related to greater awareness of the disease may facilitate the adoption of strategies aimed at the most vulnerable groups.
\end{abstract}

Key words Breast neoplasms, Programs evaluation, Risk factors
1-4 Departamento de Tecnologia de Alimentos e Saúde Pública. Universidade Federal de Mato Grosso do Sul. Cidade Universitária. s.n Caixa Postal 549. CEP: 79.070-900. Campo Grande, MS, Brasil. E-mail: abatiston@gmail.com

\section{Resumo}

Objetivos: investigar conhecimento e prática sobre os fatores de risco para o câncer de mama entre usuárias da Estratégia de Saúde da Família (ESF).

Métodos: estudo transversal com 393 mulheres com idades entre 40 e 69 anos usuárias da ESF na cidade de Dourados, MS. Realizou-se uma entrevista, por meio de um questionário semi-estruturado, investigando-se variáveis sociodemográficas, história familiar e conhecimento/prática sobre os fatores de risco para o câncer de mama. A descrição das variáveis foi feita através de frequência simples e porcentagem. A associação do conhecimento sobre os fatores de risco e as variáveis foram verificadas pelo teste exato de Fisher e qui-quadrado com nivel de significância de $5 \%$.

Resultados: a idade média foi de 52,5 $\pm 8,1$ anos, o tempo médio de estudo foi de 4,4 $\pm 3,6$ anos, $52,4 \%$ das mulheres eram pardas/negras e $66,6 \%$ possuiam companheiro. Entre as mulheres, 86,5\% receberam alguma informação sobre o câncer de mama. Os fatores de risco para a doença eram conhecidos por $54,2 \%$ das mulheres. O conhecimento sobre os fatores de risco associou-se com a história familiar $(p=0,004)$ e anos de estudo $(p=0,01)$. Diante dos fatores de risco conhecidos, a frequência de práticas preventivas entre as mulheres foi de $52,2 \%$.

Conclusões: a identificação de variáveis relacionadas a um menor conhecimento sobre a doença pode auxiliar na adoção de estratégias direcionadas aos grupos mais vulneráveis.

Palavras-chave Câncer de mama, Avaliação de programas, Fatores de risco 


\section{Introdução}

Dentre os diferentes tipos de câncer, o câncer de mama (CM) apresenta-se como um grave problema de saúde pública. É o segundo tipo de câncer mais frequente no mundo e o primeiro na população feminina. ${ }^{1}$ Apresenta altas taxas de mortalidade, afetando países com distintos níveis de desenvolvimento. ${ }^{2}$ No Brasil, as taxas de mortalidade por CM aumentaram nas últimas décadas, passando de 9,99/100.000 no ano de 1987 para 11,6/100.000 mulheres em 2007.3

O câncer de mama vem sendo amplamente estudado e, a despeito da grande quantidade de pesquisas já conduzidas sobre a patologia, sua etiologia ainda não está totalmente esclarecida, sendo a mesma atribuída a uma interação de fatores que, de certa forma, são considerados determinantes no desenvolvimento da doença. 4

Com exceção do sexo feminino, a idade é o fator de risco mais importante para o CM. A incidência da doença cresce rapidamente até os 50 anos de idade, e posteriormente, essa elevação se dá de maneira mais lenta. 5

Outros fatores como a presença dos genes $B R C A 1$ e $B R C A 2$, história familiar ou pessoal de $\mathrm{CM}$, maior densidade do tecido mamário, hiperplasia mamária atípica, exposição à radiação, fatores reprodutivos como longos períodos de história menstrual (menarca precoce e menopausa tardia), nuliparidade, primeira gestação a termo após os 30 anos de idade e ausência ou curtos períodos de amamentação também representam aumento do risco para a ocorrência do câncer de mama.6-8

No que se relaciona à prevenção da doença, especial interesse recai sobre os fatores de risco potencialmente modificáveis, como o ganho de peso após os 18 anos de idade, sobrepeso ou obesidade após a menopausa, utilização de terapias hormonais, o sedentarismo, o tabagismo e o consumo diário de bebidas alcoólicas. 8

As mudanças no estilo de vida das mulheres podem, em parte, explicar o aumento da incidência do CM, envolvendo desde o sedentarismo e hábitos alimentares até os fatores relacionados ao uso de terapias hormonais, à gestação e à amamentação, conforme já descrito. 9

O conhecimento e a compreensão acerca dos fatores de risco para o CM ganham importância à medida que alguns deles são passíveis de modificação. ${ }^{7,9}$ Deve-se considerar também, a relação entre o nível de conhecimento das mulheres e a adesão às práticas de rastreamento e detecção precoce, o que influencia diretamente o momento do diagnóstico da doença e seu prognóstico. 10
Embora a investigação do conhecimento das mulheres sobre o CM tenha sido objeto de estudos já conduzidos na população brasileira, poucos tiveram, como centralidade, o conhecimento sobre os fatores de risco para a doença.

O objetivo do presente estudo foi investigar o conhecimento e prática de mulheres de 40 a 69 anos, cadastradas na Estratégia de Saúde da Família acerca dos fatores de risco para o $\mathrm{CM}$ e a adoção de práticas preventivas relacionadas.

\section{Métodos}

Trata-se de um estudo de corte transversal, subprojeto de uma pesquisa mais ampla, ${ }^{11}$ realizada na cidade de Dourados, Estado de Mato Grosso do Sul, no período compreendido entre os meses de fevereiro e dezembro de 2008

A amostra foi composta por mulheres cadastradas na Estratégia de Saúde da Família (ESF), na faixa etária de 40 a 69 anos de idade, residentes há no mínimo 12 meses na cidade de Dourados. Foram estudadas as áreas de abrangência das 16 primeiras equipes de Saúde da Família (SF) instaladas na cidade, localizadas na área urbana, alocadas em dez Unidades Básicas de Saúde da Família (UBSF).

Para o cálculo do tamanho da amostra utilizou-se o software Raosoft ${ }^{\circledR}$ Sample Size Calculator 12 considerando-se os seguintes parâmetros: população de referência igual a 8342 mulheres; nível de confiança de $95 \%$; e erro de 5\%. O tamanho mínimo recomendado da amostra foi igual a 368 ; a este valor adicionou-se $10 \%$ para possíveis perdas, sendo assim a amostra final de 393 mulheres proporcionalmente divididas nas 16 equipes de SF estudadas.

Para a coleta dos dados realizou-se uma entrevista estruturada, utilizando-se um instrumento, previamente testado, que buscou informações sobre variáveis sociodemográficas como idade, situação conjugal, escolaridade e cor da pele. Investigou-se ainda a presença de história familiar de $\mathrm{CM}$ em parentes de $1^{\circ}$ e $2^{\circ}$ graus e o conhecimento e prática acerca dos fatores de risco para o CM e adoção de práticas para a minimização de tais fatores.

Para descrição das variáveis estudadas utilizaram-se tabelas de frequências absolutas e relativas. A associação do conhecimento sobre os fatores de risco para o $\mathrm{CM}$ e as variáveis estudadas foi verificada por meio do teste exato de Fisher e do teste qui-quadrado, admitindo-se um nível de significância de $5 \%$. Para o processamento estatístico, utilizou-se o software Epi-Info versão 3.4.3.

$\mathrm{O}$ projeto de pesquisa referente a este estudo foi 
aprovado pelo Comitê de Ética em Pesquisa da Universidade Federal de Mato Grosso do Sul sob o protocolo $\mathrm{n}^{\mathrm{o}} 1.125$.

\section{Resultados}

Entre as 393 mulheres estudadas, observou-se tempo médio de residência na cidade de Dourados de 26,4 \pm 14 anos. A idade variou de 40 a 69 anos, com média de $52,4 \pm 8,1$ anos, e 77,2\% delas apresentaram idade entre 40 e 59 anos. A maioria (87,3\%) possuía estudo formal sendo $48,6 \%$ das mulheres com um a quatro anos de estudo e tempo médio de estudo de $4,4 \pm 3,6$ anos. Em relação a outras variáveis sociodemográficas, $52,4 \%$ das mulheres relataramse da cor parda ou negra, $58,5 \%$ possuiam companheiro, $73,3 \%$ não exerciam atividade profissional fora de sua residência e $86,5 \%$ não apresentavam história familiar de câncer de mama (Tabela 1).

Quanto ao conhecimento sobre o CM, 86,5\% das mulheres informaram ter recebido algum tipo de informação sobre a doença, das quais $58 \%$ relataram que a informação foi prestada por um profissional vinculado à equipe de SF, podendo a atividade de educação em saúde ter ocorrido tanto na UBSF quanto na residência da mulher durante visitas domiciliares. Informação por meio de comunicação em massa como televisão, rádio, jornais e revistas foi referida por $28,8 \%$ das mulheres (Tabela 2).

Ao investigar-se o conhecimento especificamente sobre os fatores de risco para o $\mathrm{CM}$ observouse que $54,2 \%$ das mulheres relataram conhecer pelo menos um fator de risco (Tabela 2).

Entre as 213 mulheres que relataram conhecer algum fator de risco para o CM, 28,5\% conheciam um fator, $15,3 \%$ dois fatores, $7,6 \%$ três fatores e $2,8 \%$ quatros fatores. Os diferentes fatores de risco foram citados pelas mulheres 366 vezes. Das 366 vezes das quais os fatores de risco foram mencionados, observou-se a adoção de práticas preventivas em $52,2 \%$ dos casos (Tabela 3 ).

Houve associação entre o conhecimento de pelo menos um fator de risco para o $\mathrm{CM}$ e o número de anos de estudo e a história familiar de CM (Tabela 4).

Tabela 1

Características sociodemográficas das usuárias da Estratégia de Saúde da Família. Dourados, 2008.

\begin{tabular}{|c|c|c|}
\hline Informações sociodemográficas ( $\mathrm{N}=393)$ & $\mathbf{N}$ & $\%$ \\
\hline \multicolumn{3}{|l|}{ Idade (anos) } \\
\hline$<50$ & 160 & 40,7 \\
\hline$\geq 50$ & 233 & 59,3 \\
\hline \multicolumn{3}{|l|}{ Anos de estudo } \\
\hline Não estudou & 50 & 12,7 \\
\hline $1-4$ & 191 & 48,6 \\
\hline 5 ou mais & 152 & 38,7 \\
\hline \multicolumn{3}{|l|}{ Cor da pele } \\
\hline Branca & 186 & 47,3 \\
\hline Parda/ negra & 206 & 52,4 \\
\hline Amarela & 1 & 0,3 \\
\hline \multicolumn{3}{|l|}{ Situação conjugal } \\
\hline Com companheiro & 262 & 66,6 \\
\hline Sem companheiro & 131 & 33,3 \\
\hline \multicolumn{3}{|l|}{ Trabalha fora de casa } \\
\hline $\operatorname{Sim}$ & 105 & 26,7 \\
\hline Não & 288 & 73,3 \\
\hline \multicolumn{3}{|l|}{ Possui casa própria } \\
\hline Sim & 338 & 86,0 \\
\hline Não & 55 & 14,0 \\
\hline \multicolumn{3}{|l|}{ História familiar de câncer de mama } \\
\hline Sim & 53 & 13,5 \\
\hline Não & 340 & 86,5 \\
\hline
\end{tabular}


Tabela 2

Conhecimento dos fatores de risco para o câncer de mama pelas usuárias da Estratégia de Saúde da Família. Dourados, 2008.

\begin{tabular}{|c|c|c|}
\hline Informações sobre conhecimento & $\mathbf{N}$ & $\%$ \\
\hline \multicolumn{3}{|l|}{ Conhecimento sobre o CM $(n=393)$} \\
\hline Sim & 340 & 86,5 \\
\hline Não & 53 & 13,5 \\
\hline \multicolumn{3}{|l|}{ Fonte de informações sobre o CM $(n=340)$} \\
\hline Unidade Básica de Saúde da Família & 197 & 58,0 \\
\hline Mídia & 98 & 28,8 \\
\hline Outros meios & 36 & 10,6 \\
\hline Não responderam & 9 & 2,6 \\
\hline \multicolumn{3}{|c|}{ Conhecimento sobre fatores de risco para CM $(n=393)$} \\
\hline Sim & 213 & 54,2 \\
\hline Não & 180 & 45,8 \\
\hline
\end{tabular}

$\mathrm{CM}=$ câncer de mama.

Tabela 3

Conhecimento dos fatores de risco para o câncer de mama e hábitos preventivos praticados pelas usuárias da Estratégia de Saúde da Família. Dourados, 2008.

\begin{tabular}{|c|c|c|c|c|}
\hline \multirow[t]{2}{*}{ Fatores de risco } & \multicolumn{2}{|c|}{ Fatores de risco relatados } & \multicolumn{2}{|c|}{ Adoção de prática preventiva } \\
\hline & $\mathrm{n}$ & $\%$ & $\mathrm{n}$ & $\%$ \\
\hline Amamentação & 63 & 16,0 & 21 & 33,3 \\
\hline Tabagismo & 60 & 15,3 & 47 & 78,3 \\
\hline Não realizar consultas e exames de rotina & 45 & 11,5 & 32 & 71,1 \\
\hline Alimentação inadequada & 41 & 10,4 & 29 & 70,7 \\
\hline História familiar & 30 & 7,6 & 0 & 0,0 \\
\hline Bebidas alcoólicas & 25 & 6,4 & 22 & 88,0 \\
\hline Auto-cuidado insuficiente & 20 & 5,1 & 5 & 25,0 \\
\hline Terapia hormonal & 17 & 4,3 & 5 & 29,4 \\
\hline Traumas na mama & 16 & 4,1 & 15 & 93,8 \\
\hline Estresse & 15 & 3,8 & 6 & 40,0 \\
\hline Outros & 34 & 8,7 & 9 & 26,5 \\
\hline Total & 366 & 100,0 & 191 & 52,2 \\
\hline
\end{tabular}


Conhecimento dos fatores de risco para o câncer de mama segundo características das usuárias da Estratégia de Saúde da Família. Dourados, 2008.

\begin{tabular}{|c|c|c|c|c|c|}
\hline \multirow[t]{3}{*}{ Variáveis sociodemográficas $(\mathrm{N}=393)$} & \multicolumn{4}{|c|}{ Conhece algum fator de risco } & \multirow[t]{3}{*}{$p$} \\
\hline & \multicolumn{2}{|c|}{ Sim } & \multicolumn{2}{|c|}{ Não } & \\
\hline & $\mathrm{n}$ & $\%$ & $\mathrm{n}$ & $\%$ & \\
\hline Cor da pele ${ }^{* * *}$ & & & & & $0,41 *$ \\
\hline Branca & 99 & 46,8 & 87 & 53,2 & \\
\hline Parda/negra & 113 & 54,9 & 93 & 45,1 & \\
\hline Situação conjugal & & & & & $0,12 *$ \\
\hline Com companheiro & 148 & 56,5 & 114 & 46,5 & \\
\hline Sem Companheiro & 65 & 49,6 & 66 & 50,4 & \\
\hline Tempo de estudo (anos) & & & & & $0,01 * *$ \\
\hline Sem estudo & 18 & 36,0 & 32 & 64,0 & \\
\hline $1-4$ & 103 & 53,9 & 88 & 46,1 & \\
\hline 5 ou mais & 92 & 60,5 & 60 & 39,5 & \\
\hline História familiar de câncer de mama & & & & & $0,004 *$ \\
\hline Sim & 38 & 71,7 & 15 & 28,3 & \\
\hline Não & 175 & 51,5 & 165 & 48,5 & \\
\hline Idade (anos) & & & & & $0,73^{*}$ \\
\hline$<50$ & 88 & 55,0 & 72 & 45,0 & \\
\hline$\geq 50$ & 125 & 53,6 & 108 & 46,4 & \\
\hline
\end{tabular}

* Teste exato de Fisher; ** Teste $\chi^{2 ;}$ *** Excluída da análise uma mulher da cor amarela.

\section{Discussão}

No presente estudo, observou-se que alto percentual de mulheres possui algum conhecimento sobre o CM. Porém, aproximadamente a metade desconhece qualquer um dos fatores de risco para a doença. Este achado reforça a constatação de que o conhecimento das mulheres brasileiras sobre o CM concentra-se principalmente nos métodos para detecção precoce da doença, especialmente no auto-exame das mamas,13-15 sendo os fatores de risco pouco conhecidos por elas, bem como pouco estudados pelos pesquisadores.

Em relação ao conhecimento sobre o $\mathrm{CM}$, a maioria das mulheres relatou que a informação acerca da doença foi prestada por um profissional vinculado à equipe de SF, tanto na UBSF, como na residência da mulher durante visitas domiciliares. Observa-se que a equipe de SF possui papel relevante na disseminação de informações sobre o CM na população estudada, diferentemente dos achados de outro estudo brasileiro, 16 realizado na cidade de Goiânia, no qual apenas $15,8 \%$ das mulheres relataram um profissional da saúde como fonte de informações, sendo a mídia a principal fonte, relatada por $67,8 \%$ das mulheres, número muito superior ao encontrado neste estudo.

Embora o CM não apresente uma etiologia totalmente esclarecida, 17 muito se tem discutido sobre a existência de fatores associados a um maior risco de desenvolvimento da doença. 4

Quase metade das mulheres entrevistadas não conhece nenhum fator de risco para a doença, e entre as que possuem este conhecimento, cerca de $30 \%$ relataram conhecer apenas um fator e apenas 2,8\% conhecem quatro diferentes fatores, número máximo citado pelas mulheres. Tais resultados sugerem que os fatores de risco são pouco abordados nas ações de educação em saúde junto à população estudada.

Entre os fatores de risco estabelecidos, alguns não são passíveis de intervenção. Entre eles a idade, os fatores genéticos e a duração da história menstrual. ${ }^{8}$ Porém, existe outro grupo de fatores de risco relacionados ao comportamento e ao estilo de vida, potencialmente modificáveis, capazes de reduzir as chances de desenvolvimento do CM.18 A maioria dos fatores identificados pelas mulheres neste estudo como causadores do CM é do tipo modificável, o que favorece a adoção de práticas preventivas. 
Embora os fatores relacionados com a ausência ou curtos períodos de amamentação tenham sido os mais referidos neste estudo, apenas $16,0 \%$ das mulheres relacionaram a prática da amamentação com a redução do risco para o CM. Um estudo com dados de 47 investigações epidemiológicas em 30 países demonstrou que o risco de desenvolvimento de CM diminui com o aumento da duração da amamentação, sendo esta redução de 4,5\% para cada ano de manutenção da amamentação. 19

O efeito protetor da amamentação em relação ao $\mathrm{CM}$ não pode ser desprezado e, diferentemente de outros fatores de risco, este pode ser modificado por meio da prática do aleitamento materno. $20 \mathrm{~A}$ proteção conferida pela amamentação deve ser de conhecimento de todas as mulheres, já que isto pode aumentar a adesão a esta prática. Além disso, cabe aos profissionais de saúde encorajá-las permanentemente a amamentar seus filhos por períodos prolongados.20,21 As mulheres nulíparas por sua vez, não se beneficiam do efeito protetor da amamentação, e assim, estas devem ser estimuladas à adoção de hábitos saudáveis que minimizem os outros fatores de risco passíveis de modificação.

Outros fatores aqui citados foram o tabagismo, a não realização de consultas e exames e o tipo de alimentação.

A relação entre o fumo e o $\mathrm{CM}$ apresenta-se de forma controversa na literatura. Até recentemente, a maioria dos pesquisadores concordava que não havia provas consistentes o suficiente para determinar se o tabagismo ativo exerce papel causal no CM.22 Em estudo de revisão conduzido pelo Collaborative Group on Hormonal Factors in Breast Cancer, ${ }^{23}$ os autores analisaram 53 estudos de coorte e caso-controle e observaram que a associação entre tabagismo e CM é geralmente confundida pelo efeito do consumo de bebidas alcoólicas associado ao fumo. Nas análises realizadas com 22.225 mulheres com câncer de mama e 40.832 controles que negaram consumo de álcool, o risco de adoecer de câncer de mama em fumantes não diferiu significativamente do que em não-fumantes (risco relativo fumantes versus não-fumantes $=1,03) .23$

Contudo, em um estudo publicado recentemente, os autores realizaram uma coorte prospectiva envolvendo 79.990 mulheres com idades entre 50 e 79 anos e concluíram que seus achados sobre a relação de tabagismo ativo e CM são consistentes, tendo sido observado um aumento de $20 \%$ a $50 \%$ no risco de $\mathrm{CM}$ em associação com fatores como alta intensidade, longa duração do hábito tabágico e idade do início do tabagismo.22 Esse estudo foi corroborado pelos resultados de um painel de especialistas canadenses que revisaram a extensa lista de pesquisas publicadas mais recentemente sobre o tema e concluíram que as relações entre o fumo ativo e o $\mathrm{CM}$, tanto na pré como na pós-menopausa são consistentes com uma relação de causalidade. ${ }^{24}$

No presente trabalho as mulheres consideraram que a não realização de consultas e exames constituise um fator de risco para o CM. Esta informação sugere relevância e credibilidade conferidas ao cuidado oferecido pelos serviços de saúde, além de demonstrar a existência de um entendimento sobre a importância da adesão às ações desenvolvidas. No caso do CM, o sucesso de um programa de detecção precoce é diretamente dependente, entre outros fatores, da participação das usuárias, já que as mesmas devem comparecer às consultas, realizar os exames solicitados, participar das atividades educativas e colocar os conhecimentos adquiridos em prática.

O tipo de dieta também foi citado pelas mulheres como um fator de risco para o CM e mesmo que as evidências atuais não possibilitem a publicação de um guia que indique com segurança uma dieta para a redução do risco de CM, a American Cancer Society recomenda a adoção de um estilo de vida que promova a manutenção de um peso saudável e o consumo limitado de alimentos ricos em gorduras, especialmente as de origem animal. 25

Relacionados à dieta estão também outros fatores como o sobrepeso, a obesidade e o consumo de bebida alcoólica, sendo estes fatores de risco associados ao CM.26 Em uma recente meta-análise sobre dieta e CM, foi demonstrado que o consumo de álcool aumenta o risco de desenvolvimento de $\mathrm{CM}$ e indicam uma pequena associação inversa entre dieta saudável e risco para a doença. ${ }^{27}$

Existem outros fatores relatados na literatura como sendo fortemente associados a um maior risco de desenvolvimento do CM, como por exemplo, a idade. $5,7,8$ Todavia, este fator não foi sequer citado aqui, como também não se observou entre as mulheres entrevistadas relação significativa entre a idade e o conhecimento sobre os fatores de risco para o $\mathrm{CM}$

Já está bem estabelecido que a história familiar de CM coloca a mulher em um grupo de risco aumentado para desenvolvimento da doença, $5,7,8$ mas neste estudo este fator foi pouco citado. Uma observação que chama a atenção é o fato de que embora um número reduzido de mulheres considere a história familiar de $\mathrm{CM}$ como um fator de risco para a doença, observou-se uma relação estatisticamente significativa entre a presença de um parente com CM e maior conhecimento sobre os fatores de 
risco para a doença. Mesmo que este não seja um fator com possibilidade de modificação, é primordial que as mulheres recebam informações sobre os riscos aos quais estão expostas, pois a informação é uma grande aliada no desenvolvimento de uma atitude positiva e consciente das mulheres em relação à detecção precoce do $\mathrm{CM}$.

Algumas características sociodemográficas podem estar associadas ao nível de conhecimento das mulheres sobre os fatores de risco para o CM; além disso, a investigação dessas características pode subsidiar a identificação de fatores que as levam a uma maior participação nas ações para a detecção precoce do CM. Da mesma forma já se identificou a influência de algumas características das mulheres, como por exemplo, a cor da pele, a escolaridade e a situação conjugal na maior utilização dos serviços de saúde. 28

Ao analisar-se as características das mulheres incluídas neste estudo, não se evidenciou relação entre as variáveis cor da pele, situação conjugal e idade com o conhecimento sobre os fatores de risco para o CM. Contudo, além da história familiar anteriormente citada notou-se, como já era esperado, uma relação significativa entre anos de estudo sobre os fatores de risco, sendo que o conhecimento é maior à medida em que o nível de escolaridade aumenta, ressaltando-se assim a necessidade de projetos informativos e educativos singulares, respeitando as necessidades e limitações das usuárias.

A despeito de não estar completamente estabelecida a contribuição dos fatores de risco no desenvolvimento do $\mathrm{CM}$, reconhece-se que mudanças no estilo de vida podem acarretar benefícios importantes à saúde individual e coletiva da população. Segundo Buck, 29 um estilo de vida saudável pode ser incorporado somente quando se tem o conhecimento, a oportunidade, e a vontade para tal. Nesta lógica, ressalta-se o importante papel que os profissionais e os serviços de saúde desempenham para que informações sobre os fatores de risco para o CM sejam disseminadas, bem como a necessidade da incorporação de um estilo de vida saudável, podendo assim minimizar os riscos de desenvolvimento desta e de outros tipos de agravos crônicos não-transmissíveis. 25

Entre as mulheres entrevistadas, considera-se baixo o conhecimento sobre os fatores capazes de aumentar as chances de desenvolvimento do CM, visto o percentual de mulheres que não conhecem nenhum fator de risco para o $\mathrm{CM}$ ou que conhecem apenas um fator. Ainda mais preocupante é a constatação de que o conhecimento sobre hábitos e comportamentos de risco não são suficientes para desencadear mudanças. Isso reforça a afirmação já citada de que a adoção de um estilo de vida favorável depende não somente do conhecimento, mas também da vontade da própria mulher. ${ }^{29}$

No que tange as mulheres, é fundamental o reconhecimento de seu papel como multiplicadora de informações que possam desencadear comportamentos saudáveis em outras mulheres de sua comunidade. A educação em saúde não é tarefa fácil, principalmente se considerarmos que as mulheres assintomáticas, por não se sentirem doentes, muitas vezes ignoram os riscos aos quais estão expostas.

No que se refere aos profissionais atuantes na ESF, é fundamental que estes lancem mão de metodologias de educação que propiciem a compreensão e sensibilização da mulher para o autocuidado e para o desenvolvimento de uma atitude responsável com a sua saúde. 30

As mulheres incluídas neste estudo são cadastradas na ESF e todos os profissionais que atuam em seu cuidado foram capacitados para potencializar a detecção precoce do CM, incluindo-se as ações de educação em saúde. Contudo, em relação aos fatores de risco, essas ações precisam ser revistas e (re)planejadas a fim de que possam efetivamente, além de informar, gerar conhecimentos sólidos sobre a importância da adoção de medidas que minimizem os riscos para o desenvolvimento do CM.

Segundo Vogel, 25 os riscos devem ser reduzidos ao longo da vida da mulher. Dessa forma, os profissionais que atuam na atenção primária à saúde devem estar aptos a avaliar e quantificar os riscos individuais aos quais as mulheres estão expostas, sendo que os resultados desta avaliação devem ser claramente informados às mulheres, a fim de que este conhecimento seja propulsor de transformações no seu estilo de vida. No que é atinente aos profissionais, a avaliação do risco individualizado pode auxiliar no planejamento de ações de prevenção e rastreamento mais efetivo pela equipe de SF, considerando-se a necessidade individual de cada mulher.

Conclui-se que a maioria das mulheres possui conhecimento reduzido acerca dos fatores de risco para o $\mathrm{CM}$, e entre as mulheres que conhecem ao menos um fator de risco, a adoção de práticas preventivas para os mesmos ainda é pequena, sugerindo que o conhecimento dos fatores de risco não é suficiente para a mudança de hábitos e comportamentos.

A identificação de variáveis que relacionam-se ao maior conhecimento sobre os fatores de risco torna-se importante na medida em que estratégias 
educativas podem ser pensadas e implementadas para atender a população que se apresenta mais vulnerável em relação a um menor conhecimento sobre a doença. Embora não se possa estimar o impacto de cada um dos fatores de risco na gênese do CM, a minimização desses fatores pode, sem dúvida, contribuir para uma vida mais saudável.

\section{Referências}

1. Brasil. Ministério da Saúde. Secretaria de Atenção à Saúde. Instituto Nacional de Câncer. Coordenação de Prevenção e Vigilância de Câncer. Estimativas 2008: incidência de câncer no Brasil. Rio de Janeiro: INCA; 2007.

2. Igene $\mathrm{H}$. Global health inequalities and breast cancer: an impending public health problem for developing countries. Breast J. 2008; 14: 428-34.

3. Brasil. Ministério da Saúde. Instituto Nacional de Câncer Atlas de mortalidade por câncer. Vigilância do Câncer e fatores de risco. [Acesso em $10 \mathrm{dez} 2010$ ]. Disponível em: http://mortalidade.inca.gov.br/Mortalidade/prepararModelo 03.action

4. Pinho VFS, Coutinho ESF. Variáveis associadas ao câncer de mama em usuárias de unidades básicas de saúde. Cad Saúde Pública. 2007; 23: 1061-9.

5. Brasil. Ministério da Saúde. Instituto Nacional de Câncer. Estimativa 2010: incidência de câncer no Brasil / Instituto Nacional de Câncer. Rio de Janeiro: INCA; 2009.

6. McPherson K, Steel CM, Dixon JM. ABC of breast diseases. Breast cancer-epidemiology, risk factors, and genetics. BMJ. 2000; 321: 624-8.

7. American Cancer Society. Cancer facts \& figures 2008. Atlanta: American Cancer Society; 2008. [Acesso em 01 fev 2008]. Disponível em: http://www.cancer.org/downloads/STT/2008CAFFfinalsecured.pdf

8. American Cancer Society. Cancer facts \& figures 2010 Atlanta: American Cancer Society; 2010. [Acesso em 10 dez 2010]. Disponível em: http://www.cancer.org/acs/ groups/content/@epidemiologysurveilance/documents/doc ument/acspc-026238.pdf

9. Romieu I, Lajous M. The role of obesity, physical activityand dietary factors on the risk for breast cancer: mexican experience. Salud Pública Mex. 2009, 51 (Suppl. 2): 172-80.

10. Seah M, Tan SM. Am I breast cancer smart? Assessing breast cancer knowledge among healthcare professionals. Singapore Med J. 2007; 48: 158-62.

11. Batiston AP. Detecção precoce do câncer de mama: conhecimento e prática de mulheres e profissionais da Estratégia de Saúde da Família em Dourados/MS [tese]. Brasília: Universidade de Brasília; 2009.

12. Raosoft. Sample size calculator. [Acesso em 10 dez 2007]. Disponível em: http://www.raosoft.com/samplesize.html.

13. Freitas Júnior R, Koifman S, Santos NRM, Nunes MOA, Melo GG, Ribeiro ACG, Melo AFB. Conhecimento e prática do auto-exame de mama. Rev Assoc Med Bras. 2006; 52: $337-41$.

\section{Agradecimentos}

Agradecemos a Fundação de Apoio ao Desenvolvimento do Ensino, Ciência e Tecnologia do Estado de Mato Grosso do Sul e ao Prof. Dr. Albert Schiaveto de Souza.

14. Carelli I, Pompei LM, Mattos CS, Ferreira HG, Pescuma R, Fernandes CE, Peixoto S. Knowledge, attitude and practice of breast self-examination in a female population of metropolitan São Paulo. Breast. 2008; 17: 270-4.

15. Marinho LAB, Costa-Gurgel MS, Cecatti JG, Osis MJD. Conhecimento, atitude e prática do auto-exame das mamas em centros de saúde. Rev Saúde Pública. 2003; 37: 57682 .

16. Godinho ER, Koch HA. Fontes utilizadas pelas mulheres para aquisição de conhecimentos sobre câncer de mama. Radiol Bras. 2005; 38: 169-73.

17. Harris JR, Lippman ME, Veronesi U, Willet W. Breast Cancer. N Engl J Med. 1992; 327: 319-28.

18. Key TJ, Verkasalo PK, Banks E. Epidemiology of breast cancer. Lancet Oncol. 2001; 2: 133-40.

19. Collaborative Group on Hormonal Factors in Breast Cancer. Breast cancer and breast feeding: collaborative reanalysis of individual data from 47 epidemiological studies in 30 countries, including 50302 women with breast cancer and 96973 women without the disease. Lancet. 2002; 360: 187-95.

20. Chang-Claude J, Eby N, Kiechle M, Bastert G, Becher H. Breast feeding and breast cancer risk by age 50 among women in Germany. Cancer Causes Control. 2000; 11: 68795.

21. Silva M, Senarath U, Gunatilake M, Lokuhetty D. Prolonged breastfeeding reduces risk of breast cancer in Sri Lankan women: a case-control study. Cancer Epidemiol. 2010; 34: 267-73.

22. Luo J, Margolis KL, Wactawski-Wende J, Horn K, Messina C, Stefanick ML, Tindle HA, Tong E, Rohan TE. Association of active and passive smoking with risk of breast cancer among postmenopausal women: a prospective cohort study. BMJ. 2011; 342:d1016

23. Collaborative Group on Hormonal Factors in Breast Cancer. Alcohol, tobacco and breast cancer - collaborative reanalysis of individual data from 53 epidemiological studies, including 58515 women with breast cancer and 95 067 women without the disease. Br J Cancer. 2002; 87: 1234-45.

24. Collishaw NE, Boyd NF, Cantor KP, Hammond SK, Johnson KC, Millar J, Miller AB, Miller M, Palmer JR, Salmon AG, Turcotte F. Canadian expert panel on tobacco smoke and breast cancer risk. Ontario Tobacco Research Unit; 2009. [Acesso em 01 Abr 2011]. Disponível em: http://www.otru.org/pdf/special/expert_panel_tobacco_brea st_cancer.pdf 
25. Vogel VG. Breast cancer prevention: a review of current evidence. CA Cancer J Clin. 2000; 50: 156-70.

26. Buck K, Vrieling A, Flesch-Janys D, Chang-Claude J. Dietary patterns and the risk of postmenopausal breast cancer in a German case-control study. Cancer Causes Control. [Internet]. [Acesso em 23 dez 2010]. Disponível em: http://www.springerlink.com/content/w0463k65j94 88612/fulltext.pdf

27. Brennan SF, Cantwell MM, Cardwell CR, Velentzis LS, Woodside JV. Dietary patterns and breast cancer risk: a systematic review and meta-analysis. Am J Clin Nutr. 2010; 91: 1294-302.
28. Weinmann S, Taplin SH, Gilbert J, Beverly RK, Geiger AM, Yood MU, Mouchawar J, Manos MM, Zapka JG, Westbrook E, Barlow WE. Characteristics of women refusing follow-up for tests or symptoms suggestive of breast cancer. J Natl Cancer Inst Monogr. 2005; 35: 33-8.

29. Buck C. Después de Lalonde: Hacia la generación de salud. OPS. Boletín Epidemiol. 1986; 7: 10-6.

30. Borghesan DHP, Baraúna M, Pelloso SM, Carvalho MDB. Auto-exame das mamas: conhecimento e prática entre profissionais da área da saúde de uma instituição pública. Acta Sci., Health Sci. 2003; 25: 103-13.

Recebido em 28 de julho de 2010

Versão final apresentada em 15 de abril de 2011

Aprovado em 2 de maio de 2011 